CHRONIC OBSTRUCTIVE PULMONARY DISEASE

\title{
Should pulmonary embolism be suspected in exacerbation of chronic obstructive pulmonary disease?
}

\author{
Olivier T Rutschmann, Jacques Cornuz, Pierre-Alexandre Poletti, Pierre-Olivier Bridevaux, Olivier \\ W Hugli, Salah D Qanadli, Arnaud Perrier
}

Thorax 2007;62:121-125. doi: 10.1136/thx.2006.065557

See end of article for authors' affiliations

......................

Correspondence to: Dr O T Rutschmann, Division of General Internal Medicine, Geneva University Hospitals, 24 rue Michelidu-Crest, 1211 Geneva 14, Switzerland;

olivier.rutschmann@

houge.ch

Received 16 May 2006

Accepted 6 October 2006

Published Online First

13 November 2006

\begin{abstract}
Background: The cause of acute exacerbation of chronic obstructive pulmonary disease (COPD) is often difficult to determine. Pulmonary embolism may be a trigger of acute dyspnoea in patients with COPD. Aim: To determine the prevalence of pulmonary embolism in patients with acute exacerbation of COPD. Methods: 123 consecutive patients admitted to the emergency departments of two academic teaching hospitals for acute exacerbation of moderate to very severe COPD were included. Pulmonary embolism was investigated in all patients (whether or not clinically suspected) following a standardised algorithm based on D-dimer testing, lower-limb venous ultrasonography and multidetector helical computed tomography scan. Results: Pulmonary embolism was ruled out by a D-dimer value $<500 \mu \mathrm{g} / \mathrm{l}$ in $28(23 \%)$ patients and a by negative chest computed tomography scan in 91 (74\%). Computed tomography scan showed pulmonary embolism in four patients $(3.3 \%, 95 \%$ confidence interval $(\mathrm{Cl}), 1.2 \%$ to $8 \%$ ), including three lobar and one sub-segmental embolisms. The prevalence of pulmonary embolism was $6.2 \%(\mathrm{n}=3 ; 95 \% \mathrm{Cl}, 2.3 \%$ to $16.9 \%$ ) in the 48 patients who had a clinical suspicion of pulmonary embolism and $1.3 \%(n=1 ; 95 \% \mathrm{Cl}, 0.3 \%$ to $7.1 \%$ ) in those not suspected. In two cases with positive computed tomography scan, the venous ultrasonography also showed a proximal deep-vein thrombosis. No other patient was diagnosed with venous thrombosis.

Conclusions: The prevalence of unsuspected pulmonary embolism is very low in patients admitted in the emergency department for an acute exacerbation of their COPD. These results argue against a systematic examination for pulmonary embolism in this population.
\end{abstract}

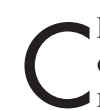
hronic obstructive pulmonary disease (COPD) is a leading cause of morbidity and mortality worldwide, and represents a huge economical burden for the healthcare system. ${ }^{12}$ Acute exacerbation of COPD is a frequent reason for visit to the emergency department. ${ }^{3}$ The most common triggers of exacerbations are infections of the tracheobronchial tree ${ }^{5}$ and air pollution, ${ }^{6}$ but the cause of exacerbation is impossible to determine in up to $30 \%$ of cases. ${ }^{7}$

Clinical presentation of COPD exacerbation includes worsening of dyspnoea, increased cough and sputum, and changes in the aspect of expectorations. Although clinical criteria have been used to determine which patient should be treated with antibiotics, ${ }^{8}$ these criteria are neither sensitive nor specific enough to exclude other causes of dyspnoea in this population. Other frequent clinical conditions may mimic the symptoms of COPD exacerbation, including congestive heart failure, pneumonia, pneumothorax, pleural effusion and pulmonary embolism. ${ }^{7}$

On the other hand, COPD is often cited among the risk factors for acute venous thromboembolism and was recently identified as an independent predictor of pulmonary embolism. ${ }^{9}$ In a small series of patients, the prevalence of deep vein thrombosis in patients admitted with acute exacerbation of COPD was $31 \% .{ }^{111}$ Similarly, on the basis of ventilationperfusion lung scintigraphy, the prevalence of pulmonary embolism in patients admitted with acute exacerbation of COPD was as high as $20 \% .{ }^{12}{ }^{13}$ More recently, Tillie-Leblond et $a l^{14}$ explored the prevalence of pulmonary embolism in a cohort of patients with COPD with unexplained dyspnoea and found a rate of $25 \%$ in this population. However, that study was performed in a highly selected subgroup of patients, and does not resolve the dilemma that primary care and emergency doctors face when taking care of acute exacerbation of COPD.
Our prospective study aimed to determine the prevalence of pulmonary embolism in patients presenting to the emergency department with acute exacerbation of COPD, using a validated diagnostic strategy based on the use of D-dimer, lower limb ultrasonography and multidetector computed tomography. ${ }^{15}$

\section{MATERIALS AND METHODS}

\section{Study design and setting}

This was a cross-sectional study performed in the emergency departments of two general teaching hospitals in Geneva and Lausanne, Switzerland, admitting 60000 and 35000 patients/ year, respectively.

\section{Study population}

All consecutive adult patients having moderate to very severe COPD according to the global initiative for chronic obstructive lung disease definition ${ }^{7}$ and admitted for an acute exacerbation of their COPD were eligible for the study. COPD had to be confirmed by pulmonary testing during the index admission. Exacerbation was defined as any worsening of dyspnoea sufficiently severe to warrant an admission to the emergency room. Patients with renal failure (plasma creatinin $\geqslant 150 \mu \mathrm{mol} /$ 1), allergy to intravenous contrast medium, on long-term anticoagulation therapy at admission or in respiratory distress requiring intubation/non-invasive ventilation were excluded. Patients with an obvious alternative cause of dyspnoea (lobar pneumonia, pneumothorax, pulmonary oedema and other obvious causes) were also excluded.

\section{Abbreviation: COPD, chronic obstructive pulmonary disease}




\section{Study protocol}

Every patient was evaluated by the emergency room physician with a standardised protocol including clinical evaluation, chest $x$ ray, electrocardiogram and arterial blood gas analysis. For each patient, the clinician had to determine whether pulmonary embolism could be clinically suspected or not. No suspicion of pulmonary embolism was defined as a patient in whom the physician-in-charge would not have examined for a pulmonary embolism outside the study. After the initial clinical evaluation, pulmonary embolism was investigated in all included patients following a standardised validated algorithm based on D-dimer (rapid enzyme-linked immunosorbent assay, Vidas-DD Exclusion, BioMérieux, Marcy L'Etoile, France) measurement, lower-limb ultrasonography and multidetector helical computed tomography scan..$^{15}$ A plasma D-dimer value $<500 \mu \mathrm{g} / \mathrm{l}$ ruled out pulmonary embolism, and no further examination was performed. ${ }^{15}{ }^{16}$ For patients with a D-dimer value $>500 \mu \mathrm{g} / \mathrm{l}$, both the lower-limb compression ultrasonography of the proximal veins and thoracic multidetector helical computed tomography scan were performed according to a previously published algorithm. ${ }^{15}$

Ultrasonographic criteria for deep-vein thrombosis were noncompressibility or incomplete compressibility of the vein. ${ }^{17}$ Computed tomography scans were obtained using a 16-slice multidetector-row computed tomography. These examinations were performed in breath hold, with the injection of $100 \mathrm{ml}$ of non-ionic contrast material (iopromide; Ultravist-300R, Schering, Baas, Switzerland), with a power injector at $3 \mathrm{ml} / \mathrm{s}$, using a slice thickness of $1.5 \mathrm{~mm}$, tube voltage of $120 \mathrm{kV}$ and tube current of $200 \mathrm{~mA}$. In patients with a body mass index of $>30 \mathrm{~kg} / \mathrm{m}^{2}$, the volume of contrast material was increased to $120 \mathrm{ml}$ and the tube voltage to $140 \mathrm{kV}$. Pulmonary embolism was diagnosed if contrast material outlined an intraluminal defect, or if a vessel was totally occluded by low-attenuation material. ${ }^{15}$

\section{Outcomes}

The main outcome measure was the proportion of patients diagnosed with pulmonary embolism, globally and in the subgroups of patients with or without clinical suspicion of pulmonary embolism. These groups were compared in terms of demographic characteristics and clinical presentation using $\chi^{2}$ or Fisher's exact tests for categorical and t tests for continuous data (SPSS V.11.0 for windows).

The full study design was reported previously. ${ }^{18}$

\section{Ethics}

The protocol was approved by our institutional review board, and each patient gave his or her written informed consent to participate.

\section{RESULTS}

In all, 521 patients were screened for inclusion between 11 February 2003 and 17 December 2004. A total of 385 patients were excluded after the initial screening. Figure 1 summarises the reasons for exclusion. Thus, 136 patients were initially included. Thirteen patients were further excluded: eleven because COPD was not confirmed on spirometry and two because computed tomography scan was not performed. Table 1 shows the characteristics of the 123 included patients.

On the basis of their initial clinical evaluation, the emergency department physicians suspected pulmonary embolism in 48 (39\%) patients and did not suspect it in $75(61 \%)$ patients. No statistically significant differences were seen between both groups of patients in terms of age groups, sex ratio, pulmonary embolism risk factors, chest $x$ ray and electrocardiogram findings (data not shown). Few differences were observed in the clinical presentation: chest pain was present in $42 \%$ of the patients with a clinical suspicion of pulmonary embolism, compared with $19 \%$ of the patients without suspicion $(\mathrm{p}=0.008)$, but the pain characteristics were similar in both groups. Cough was less frequent when pulmonary embolism was suspected $(75 \% \vee 93 \%, p=0.009)$. Similarly, sputum was less frequent when pulmonary embolism was suspected (54\% v $77 \%, \mathrm{p}=0.016$ ), but sputum characteristics were similar in both groups when sputum was present. Syncope was the presenting complaint in $3(6 \%)$ patients with clinical suspicion

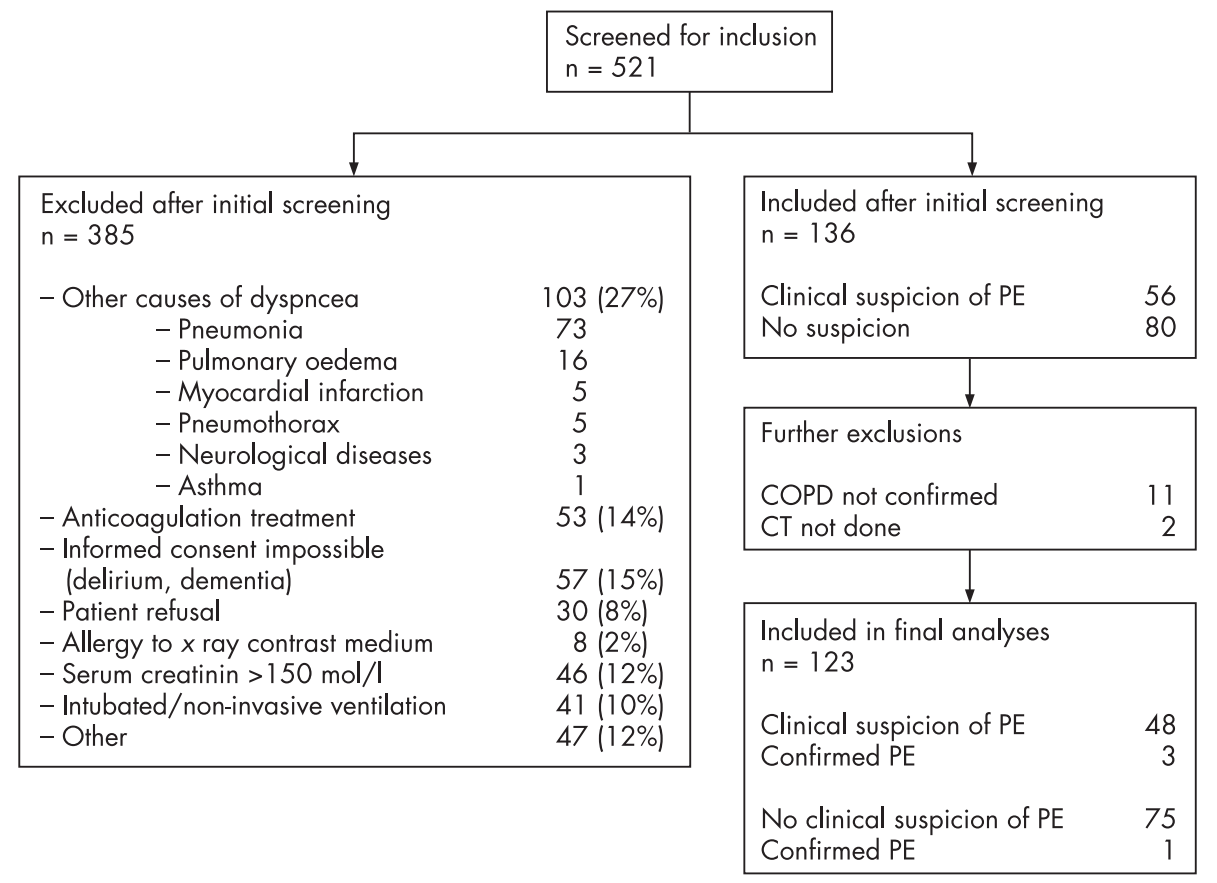

Figure 1 Study flowchart summarising inclusion and exclusion processes and main outcomes. COPD, chronic obstructive pulmonary disease; CT, computed tomography; $\mathrm{PE}$, pulmonary embolism. 
Table 1 Characteristics of patients $(n=123)$ with acute exacerbation of their chronic obstructive pulmonary disease

\begin{tabular}{|c|c|}
\hline Characteristics & $n=123$ \\
\hline Age (years), mean (SD) & $71(8)$ \\
\hline Women & $39(32 \%)$ \\
\hline \multicolumn{2}{|l|}{ Severity of COPD, mean (SD) FEVI } \\
\hline Very severe, $0.65(15)$ I & $35(28 \%)$ \\
\hline Severe, $(0.92(0.26) I$ & $61(50 \%)$ \\
\hline Moderate, $(1.42(0.38) \mathrm{I}$ & $27(22 \%)$ \\
\hline \multicolumn{2}{|l|}{ Risk factors } \\
\hline Family history of deep vein thrombosis or $\mathrm{PE}$ & $11(9 \%)$ \\
\hline Previous deep vein thrombosis or PE & $5(3 \%)$ \\
\hline Known heart failure & $15(12 \%)$ \\
\hline Paralysis & $4(3 \%)$ \\
\hline Cancer & $6(5 \%)$ \\
\hline Surgery within 1 month & $2(2 \%)$ \\
\hline Immobilisation & $20(16 \%)$ \\
\hline Hormone replacement therapy & $5(4 \%)$ \\
\hline \multicolumn{2}{|l|}{ Other comorbid conditions } \\
\hline Hypertension & $41(33 \%)$ \\
\hline Diabetes & $8(7 \%)$ \\
\hline Ischaemic heart disease & $19(15 \%)$ \\
\hline \multicolumn{2}{|l|}{ Clinical presentation } \\
\hline Chest pain & $34(28 \%)$ \\
\hline Pleuritic & $17(50 \%)$ \\
\hline Oppressive & $7(6 \%)$ \\
\hline Reproduced by palpation & $2(2 \%)$ \\
\hline Others non-pleuritic & $8(7 \%)$ \\
\hline Cough & $106(86 \%)$ \\
\hline Increased frequency & $79(64 \%)$ \\
\hline Sputum & $84(68 \%)$ \\
\hline Increased volume & $63(51 \%)$ \\
\hline Purulent & $50(41 \%)$ \\
\hline Syncope & $3(2 \%)$ \\
\hline Orthopnoea & $44(36 \%)$ \\
\hline Leg pain & $7(6 \%)$ \\
\hline \multicolumn{2}{|l|}{ Physical examination } \\
\hline Heart rate (beats/min), mean (SD) & $99(21)$ \\
\hline Respiratory rate (breaths $/ \mathrm{min})$, mean $(\mathrm{SD})$ & $26(6)$ \\
\hline Systolic blood pressure (mm Hg), mean (SD) & $150(28)$ \\
\hline Diastolic blood pressure (mm Hg), mean (SD) & $83(17)$ \\
\hline Temperature $\left({ }^{\circ} \mathrm{C}\right)$, mean $(\mathrm{SD})$ & $37.1(0.8)$ \\
\hline Lung rales & $60(49 \%)$ \\
\hline Turgescent jugular vein & $13(11 \%)$ \\
\hline Signs of deep vein thrombosis & $1(1 \%)$ \\
\hline Signs of chronic venous insufficiency & $25(21 \%)$ \\
\hline \multicolumn{2}{|l|}{ Blood gas analyses } \\
\hline $\mathrm{PH}$, mean $(\mathrm{SD})$ & $7.43(0.06)$ \\
\hline $\mathrm{PaO}_{2}(\mathrm{kPa})$, mean $(\mathrm{SD})$ & $10.1(6.3)$ \\
\hline $\mathrm{PaCO}_{2}(\mathrm{kPa})$, mean $(\mathrm{SD})$ & $6.1(1.6)$ \\
\hline \multicolumn{2}{|l|}{ Chest $x$ ray } \\
\hline Elevated hemidiaphragm & $6(5 \%)$ \\
\hline Band atelectasis & $10(8 \%)$ \\
\hline Cardiomegaly & $19(15 \%)$ \\
\hline Infiltrate (non-lobar) & $21(17 \%)$ \\
\hline Signs of heart failure & $4(3 \%)$ \\
\hline $\begin{array}{l}\text { Abnormal ECG (right bundle branch/negative T wave/ } \\
\text { S1Q3) }\end{array}$ & $21(17 \%)$ \\
\hline
\end{tabular}

COPD, chronic obstructive pulmonary disease; ECG, electrocardiogram, $\mathrm{FEV}$, forced expiratory volume in one second; $\mathrm{PaCO}_{2}$, arterial carbon dioxide tension; $\mathrm{PaO}_{2}$, alveolar oxygen tension; $\mathrm{PE}$, pulmonary embolism.

of pulmonary embolism and in none of the remaining patients $(\mathrm{p}=0.051)$. Finally, severe hypoxaemia was more frequent in patients suspected of having pulmonary embolism: $48 \%$ of the patients suspected of having pulmonary embolism had arterial partial pressure of oxygen values $<8 \mathrm{kPa}$ compared with $20 \%$ in the group of patients not suspected of having pulmonary embolism $(p=0.003)$.

D-dimer value was obtained for all patients. Pulmonary embolism was excluded on the basis of normal $(<500 \mu \mathrm{g} / \mathrm{l})$ D-dimer levels in $28(23 \%)$ patients (table 2 ).

The percentages of negative D-dimer $(<500 \mu \mathrm{g} / \mathrm{l})$ were similar in patients with and in those without a clinical suspicion of pulmonary embolism. Computed tomography scan
Table 2 Results of D-dimer, lower-limb ultrasonography and chest computed tomography scan in 48 patients with a clinical suspicion of pulmonary embolism and in 75 patients without clinical suspicion of pulmonary embolism

\begin{tabular}{|c|c|c|c|}
\hline & $\begin{array}{l}\text { Patients with } \\
\text { suspicion of } \\
\text { pulmonary } \\
\text { embolism, n (\%) }\end{array}$ & $\begin{array}{l}\text { Patients without } \\
\text { a suspicion of } \\
\text { pulmonary } \\
\text { embolism, n (\%) }\end{array}$ & $\begin{array}{l}\text { p Value } \\
\text { (suspected v not } \\
\text { suspected) }\end{array}$ \\
\hline$n$ & $48(39 \%)$ & $75(61 \%)$ & \\
\hline D-dimer $<500 \mu \mathrm{g} / \mathrm{l}$ & $11(23 \%)$ & $17(23 \%)$ & 0.97 \\
\hline $\begin{array}{l}\text { Positive } \\
\text { ultrasonography* }\end{array}$ & $1(2.1 \%)$ & $1(1.3 \%)$ & 0.63 \\
\hline $\begin{array}{l}\text { Positive computed } \\
\text { tomography scan }\end{array}$ & $3(6.3 \%)$ & $1(1.3 \%)$ & 0.13 \\
\hline
\end{tabular}

of the lung was performed in $95(77 \%)$ patients and lower-limb ultrasonography in $90(73 \%)$ patients. In $5(4 \%)$ patients, venous ultrasonography could not be performed for practical reasons.

Computed tomography scan of the lungs showed pulmonary embolism in $4(3.3 \%)$ patients (95\% CI $1.3 \%$ to $8.0 \%)$, including three lobar and one subsegmental pulmonary embolism. In two cases, the lower limb ultrasonography also showed a proximal deep-vein thrombosis. None of the patients with a negative computed tomography scan was diagnosed with deep-vein thrombosis in lower limb ultrasonography. Pulmonary embolism was diagnosed in $3(6.2 \%)$ patients (95\% CI $2.3 \%$ to $16.9 \%$ ) who had a clinical suspicion of pulmonary embolism and in 1 ( $1.3 \%$ ) patient $(95 \%$ CI $0.3 \%$ to $7.1 \%$ ) who had no suspicion (table 2).

\section{DISCUSSION}

In this prospective series of 123 patients with moderate to severe COPD admitted with worsened dyspnoea, we found that the prevalence of pulmonary embolism was very low. Indeed, a diagnostic procedure based on systematic D-dimer testing, lower-limb ultrasonography and computed tomography scan of the lungs identified pulmonary embolism in only $4(3 \%)$ patients (95\% CI $1 \%$ to $8 \%$ ). This prevalence was even lower in the subgroup of patients without a clinical suspicion of pulmonary embolism in the emergency room. In this subgroup, only one pulmonary embolism was diagnosed by our systematic examination (prevalence 1.3\%). The three remaining cases were suspected by the emergency doctor and would have been diagnosed outside the study.

These results contrast strikingly with studies suggesting that the prevalence of pulmonary embolism might be as high as 15$25 \%$. These differences may have different explanations. First, the imaging method used for diagnosing pulmonary embolism is crucial in a population of patients with chronic lung diseases. Ventilation-perfusion scintigraphy was used in previous studies. ${ }^{12}{ }^{13}$ Although scintigraphy is an acceptable tool for the diagnosis of pulmonary embolism in patients without underlying lung diseases, the interpretation of lung scintigraphy is difficult in patients with COPD, which might have led to an overestimation of the true prevalence of pulmonary embolism in that population. ${ }^{19}$ In contrast, the performance of computed tomography scan is not altered in patients with COPD. ${ }^{19}{ }^{20} \mathrm{We}$ used a validated algorithm based on multidetector computed tomography scan, ${ }^{15}$ which should have minimised the risk of false-positive findings.

Secondly, most studies exploring the relationship between COPD exacerbation and deep-vein thrombosis or pulmonary 
embolism were retrospective or were based on autopsy findings. ${ }^{9122}$ Our prospective design and our inclusion criteria limited selection biases.

Thirdly and most importantly, we studied an unselected population of patients admitted to the emergency room of two general teaching hospitals with an acute exacerbation of their COPD. Some studies included only the most severely ill patients admitted to intensive care units. ${ }^{11}$ Others excluded patients with normal D-dimer values. ${ }^{13}$ In our study, $23 \%$ of the patients had D-dimer values $<500 \mu \mathrm{g} / \mathrm{l}$ excluding pulmonary embolism. Excluding these patients would overestimate the true prevalence of pulmonary embolism in an unselected population of patients with COPD with acute exacerbation.

Nevertheless, none of those explanations account for the striking difference between our findings and the $25 \%$ prevalence of pulmonary embolism recently reported by TillieLeblond et al. ${ }^{14}$ That series was prospective and used multidetector computed tomography. The inclusion criteria seemed similar to those of our study and are summarised as exacerbation of COPD of unknown origin. However, their criteria for lower respiratory infection were broad, and they excluded patients with increased sputum volume and/or increased sputum purulence, fever, history of cold and sore throat. We included those patients, except when the initial $x$ ray showed obvious signs of pneumonia. This might account for part of the difference in pulmonary embolism prevalence compared with our series. However, the gap remains large, and we feel that the main difference was truly the clinical setting and patient selection. The French study was conducted in a large referral inpatient respiratory disease department, whereas our population was representative of the patients treated by emergency physicians in general hospitals. Furthermore, 29\% of the patients with COPD in the French study had cancer compared with only $5 \%$ in our series.

Our study has some limitations. Firstly, the number of excluded patients was relatively high. But $41 \%$ of those patients were excluded for reasons unlikely to have introduced bias (refusal or inability to consent, ongoing oral anticoagulant treatment, allergy or renal failure). Patients who were excluded because exacerbation of their dyspnoea was attributed to another cause had very clear alternative diagnoses. In particular, pneumonia was defined by both a consolidation on chest $x$ ray and a compatible clinical presentation (fever and purulent sputum). Therefore, only $10 \%$ of the patients were excluded because they required non-invasive ventilation or intubation and who were not able to undergo a computed tomography scan. Whether the prevalence in this subgroup could be higher as suggested by Schonhofer and Kohler ${ }^{11}$ is unknown. Secondly, the small number of cases of pulmonary embolism did not allow us to explore whether clinical or biological characteristics could identify a subgroup of patients with a higher prevalence of pulmonary embolism. Despite this limitation and although this was not a primary objective of this study, we were able to identify some clinical characteristics that led the emergency physician to suspect pulmonary embolism. Pulmonary embolism was more frequently suspected in the presence of chest pain, in the absence of purulent sputum or in the presence of a severe hypoxaemia.

In conclusion, our results stand against a high prevalence of unsuspected pulmonary embolism in patients admitted for an acute exacerbation of their COPD and against a systematic examination for pulmonary embolism in these patients. However, further studies are needed to confirm our findings in similar groups of patients. When pulmonary embolism is clinically suspected, a diagnostic strategy based on D-dimer testing, lower-limb ultrasonography and multidetector computed tomography scan is appropriate.

\section{ACKNOWLEDGEMENTS}

We thank Louise Riberdy who collected the data in Geneva.

\section{Authors' affiliations}

Olivier T Rutschmann, Arnaud Perrier, Division of General Internal Medicine, Department of Medicine, Geneva University Hospital and Faculty of Medicine, Geneva, Switzerland

Jacques Cornuz, Pierre-Olivier Bridevaux, Policlinique médicale universitaire, Centre Hospitalier Universitaire Vaudois (CHUV), Lausanne, Switzerland

Pierre-Alexandre Poletti, Division of Radiodiagnosis and Interventional Radiology, Department of Medical Radiology and Informatics, Geneva University Hospital and Faculty of Medicine, Geneva, Switzerland

Olivier W Hugli, Emergency Department, Centre Hospitalier Universitaire Vaudois (CHUV), Lausanne, Switzerland

Salah D Qanadli, Cardiovascular and Metabolic Diseases Centre,

Department of Radiology, Centre Hospitalier Universitaire Vaudois (CHUV), Lausanne, Switzerland

Funding: This project was supported by the Swiss National Fund for Scientific Research Grant \# 3200-068146. The researchers are independent from funders.

Competing interests: None declared.

\section{REFERENCES}

1 Mannino DM, Homa DM, Akinbami $\sqcup$, et al. Chronic obstructive pulmonary disease surveillance-United States, 1971-2000. Respir Care 2002;47:1 184-99.

2 Mannino DM, Homa DM, Akinbami $\sqcup$, et al. Chronic obstructive pulmonary disease surveillance-United States, 1971-2000. MMWR CDC Surveill Summ 2002;51:1-16.

3 Camargo CA Jr, Roberts J, Clark S. US emergency department visits for COPD exacerbations between 1992 and 1998. Am J Epidemiol 2001;153:S80.

4 Soler N, Torres A, Ewig S, et al. Bronchial microbial patterns in severe exacerbations of chronic obstructive pulmonary disease (COPD) requiring mechanical ventilation. Am J Respir Crit Care Med 1998;157:1498-505.

5 Wilson R. Evidence of bacterial infection in acute exacerbations of chronic bronchitis. Semin Respir Infect 2000;15:208-15

6 Stockley RA, O'Brien C, Pye A, et al. Relationship of sputum color to nature and outpatient management of acute exacerbations of COPD. Chest 2000; 117:1638-45.

7 Pauwels RA, Buist AS, Calverley PM, et al. Global strategy for the diagnosis, management, and prevention of chronic obstructive pulmonary disease. NHLBI/ WHO global initiative for chronic obstructive lung disease (GOLD) workshop summary. Am J Respir Crit Care Med 2001;163:1256-76.

8 Anthonisen NR, Manfreda J, Warren CP, et al. Antibiotic therapy in exacerbations of chronic obstructive pulmonary disease. Ann Intern Med 1987; 106:196-204.

9 Poulsen SH, Noer I, Moller JE, et al. Clinical outcome of patients with suspected pulmonary embolism. A follow-up study of 588 consecutive patients. J Intern Med 2001;250:137-43.

10 Winter JH, Buckler PW, Bautista AP, et al. Frequency of venous thrombosis in patients with an exacerbation of chronic obstructive lung disease. Thorax 1983:38:605-8.

11 Schonhofer B, Kohler D. Prevalence of deep-vein thrombosis of the leg in patients with acute exacerbation of chronic obstructive pulmonary disease. Respiration 1998:65:173-7.

12 Erelel M, Cuhadaroglu C, Ece T, et al. The frequency of deep venous thrombosis and pulmonary embolus in acute exacerbation of chronic obstructive pulmonary disease. Respir Med 2002;96:515-18.

13 Mispelaere D, Glerant JC, Audebert M, et al. Pulmonary embolism and sibilant types of chronic obstructive pulmonary disease decompensations. Rev Mal Respir 2002;19:415-23.

14 Tillie-Leblond I, Marquette $\mathrm{CH}$, Perez T, et al. Pulmonary embolism in patients with unexplained exacerbation of chronic obstructive pulmonary disease: prevalence and risk factors. Ann Intern Med 2006;144:390-6.

15 Perrier A, Roy PM, Sanchez O, et al. Multidetector-row computed tomography in suspected pulmonary embolism. N Engl J Med 2005;352:1760-8.

16 Sohne M, Kruip MJ, Nijkeuter M, et al. Accuracy of clinical decision rule, D-dimer and spiral computed tomography in patients with malignancy, previous venous thromboembolism, COPD or heart failure and in older patients with suspected pulmonary embolism. J Thromb Haemost 2006;4:1042-6.

17 Lensing AW, Prandoni P, Brandjes D, et al. Detection of deep-vein thrombosis by real-time b-mode ultrasonography. N Engl J Med 1989;320:342-5.

18 Perrier A, Perneger T, Cornuz J, et al. The COPD-PE study: prevalence and prediction of pulmonary embolism in acute exacerbations of chronic obstructive pulmonary disease. Rev Mal Respir 2004;21:791-6.

19 Hartmann IJ, Hagen PJ, Melissant CF, et al. Diagnosing acute pulmonary embolism: effect of chronic obstructive pulmonary disease on the performance of D-dimer testing, ventilation/perfusion scintigraphy, spiral computed tomographic angiography, and conventional angiography. Antelope study group. Advances 
in new technologies evaluating the localization of pulmonary embolism. Am J Respir Critic Care Med 2000;162:2232-7.

20 Remy-Jardin M, Tillie-Leblond I, Szapiro D, et al. CT angiography of pulmonary embolism in patients with underlying respiratory disease: impact of multislice CT on image quality and negative predictive value. Eur Radiol 2002;12:1971-8.
21 Janssens JP, Herrmann F, MacGee W et al Cause of death in older patients with anatomo-pathological evidence of chronic bronchitis or emphysema: a case-control study based on autopsy findings. J Am Geriatr Soc 2001;49:571-6.

22 Pineda LA, Hathwar VS, Grant BJ. Clinical suspicion of fatal pulmonary embolism. Chest 2001;120:791-5.

\section{LUNG ALERT}

Reduced lung function at birth may be a risk factor for later asthma

$\Delta$ Håland G, Lødrup Carlsen KC, Sandvik L, et al. Reduced lung function at birth and the risk of asthma at 10 years of age. N Engl J Med 2006;355:1682-9

A wheeze during childhood may be related to the development of asthma in later life, especially if it is a "transient early wheeze". The authors have studied whether lung function abnormalities in a child shortly after birth could be used as a predictor of asthma at age 10 .

The lung function of 802 newborns was measured (mean age 2.7; SD 0.9 days) using a pneumotachograph with a face mask during quiet tidal breathing. These data were used to calculate peak tidal expiratory flow/total expiratory time $\left(\mathrm{T}_{\mathrm{ptef}} / \mathrm{T}_{\mathrm{e}}\right)$ and respiratory-system compliance.

At 10 years the children were assessed for a diagnosis of asthma based either on history, previous diagnosis, hyperresponsiveness, treadmill induced bronchoconstriction and/or clinical examination. At this time 616 (77\%) remained under follow-up; 2 were excluded on the basis of insufficient data.

$\mathrm{T}_{\text {ptef }} / \mathrm{T}_{\mathrm{e}}<0.2$ or the median and respiratory-system compliance less than the median were used as possible predictors of asthma. Results show that lung function abnormalities have a low positive predictive value for asthma at 10 years, but may be a risk factor for later childhood asthma.

Adjustments were made for intrauterine smoke exposure, parental asthma, rhinoconjunctivitis and sex. However, any adjustment for environmental factors and ethnic differences were not made clear. Also, the diagnostic criteria for asthma were changeable depending on variations in the definition of wheeze or the threshold for starting asthma treatment.

This was an interesting study that makes clear the highly variable nature of asthma and airway development in early childhood. Further study will be required in order to assess whether lung function testing, in combination with other assessment tools of airway development in early child, will provide enough information to influence early intervention with children deemed to be at risk of developing asthma.

Raymond Ramnarine

FY1 Respiratory Medicine, Homerton University Hospital; raymondr@doctors.org.uk 